\title{
The occupational risk of Helicobacter pylori infection among gastroenterologists and their assistants
}

\author{
Claudia Peters ${ }^{1 *}$, Anja Schablon ${ }^{1}$, Melanie Harling ${ }^{1}$, Claudia Wohlert ${ }^{1},{\text { José Torres } \text { Costa }^{2} \text { and Albert Nienhaus }}^{1}$
}

\begin{abstract}
Background: Helicobacter pylori is a widely spread bacterium that mainly inhabits the gastric mucosa and can lead to serious illnesses such as peptic ulcer disease, gastric carcinoma and gastric MALT lymphoma. The oral-oral route seems to be the main transmission route. The fact that endoscopes are contaminated after being used to perform a gastroscopy leads one to question whether gastroenterologists and endoscopy nurses and assistants run a higher risk of infection.
\end{abstract}

Methods: A systematic search for literature was conducted in the MEDLINE and EMBASE databases and further publications were found in reference lists of relevant articles. Epidemiological studies on the occupational exposure of endoscopy personnel were collected and their quality was assessed. Pooled effect estimates were identified in a meta-analysis.

Results: Of the 24 studies included in the analysis, 15 were considered to be methodologically good. Of these 15 studies, eight single studies showed a statistically significant increased risk of infection for gastroenterologists, and five for their assistants. Meta-analysis across all methodologically good studies found a statistically significant risk of 1.6 (95\% Cl 1.3-2.0) for doctors. The pooled effect estimates also indicated a statistically significant risk of Helicobacter pylori infection (RR 1.4; 95\%Cl 1.1-1.8) for assistants too.

When studies are stratified by medical and non-medical control groups, statistically significant risks can only be recognised in the comparison with non-medical controls.

Conclusions: In summary, our results demonstrated an increased risk of Helicobacter pylori infection among gastroenterological personnel. However, the choice of control group is important for making a valid assessment of occupational exposure risks.

\section{Background}

Helicobacter pylori (H. pylori) is a gram-negative, spiralshaped bacterium that mainly inhabits and multiplies in the gastric mucosa $[1,2]$. The bacterium produces the enzyme urease and this convert urea into carbon dioxide and ammonia. The ammonium envelope enables it to resist the acidic environment in the stomach [1]. The prevalence of $H$. pylori varies widely from region to region, with an especially marked difference between industrial and developing countries. The estimated prevalence in Asia is 50 to $80 \%, 30$ to $50 \%$ in Western

\footnotetext{
* Correspondence: c.peters@uke.de

'University Medical Center Hamburg-Eppendorf, Institute for Health Service Research in Dermatology and Nursing, Hamburg, Germany

Full list of author information is available at the end of the article
}

Europe and 30\% in North America [3]. In Germany, the prevalence of $H$. pylori infections among the population is between 20 and 70\% [4]. Within the framework of the German National Health Inverview and Examination Survey 1998 a $40 \%$ prevalence of $H$. pylori antibodies among the general population was found. In the youngest age group (aged 18 to 29 ) the prevalence was $22 \%$, while in the oldest age group it was $61 \%$. In the low socioeconomic status group, the prevalence was $51 \%$, whereas it was $29 \%$ in the upper class [4]. There is a positive correlation between the number of persons in a household and the rate of $H$. pylori infection [5].

H. pylori is associated with peptic ulcer disease, distal gastric carcinoma and gastric MALT lymphoma [1]. It has long been known that the stomach harbours a

\section{Biomed Central}

(c) 2011 Peters et al; licensee BioMed Central Ltd. This is an Open Access article distributed under the terms of the Creative Commons Attribution License (http://creativecommons.org/licenses/by/2.0), which permits unrestricted use, distribution, and reproduction in any medium, provided the original work is properly cited. 
population of bacteria [1], but not until Marshall and Warren's work in 1984 [6] was the link between Campylobacter pyloridis, as $H$. pylori was then known, and chronic gastritis appreciated, which "revolutionised the understanding of pathological gastric processes" [1]. Individuals infected with $H$. pylori run a three times greater risk of contracting an ulcus ventriculi and a two and a half times greater risk of developing adenocarcinoma of the stomach [7]. There also appears to be a synergistic carcinogenic effect when smoking and $H$. pylori infections are combined [8].

The transmission routes of $H$. pylori have yet to be fully explained $[1,4,6]$. The likelihood of infection increases with low social status and the associated crowded living conditions. The infection is mainly acquired in childhood [9]. H. pylori has been isolated from faeces [10], gastric juice, vomit, saliva and dental plaque $[11,12]$. It is transmitted from person to person. The oral-oral route seems to be the main route of transmission. Contact with regurgitated matter seems to play a more important role in transmission than contact with saliva, since promiscuity is not a transmission risk factor $[2,7]$ and transmission between married couples is rare [13]. Dentists come into close contact with their patients' saliva, but although they have been found to be at greater risk [14], there is insufficient evidence of an increased risk of infection $[15,16]$. There are reports of a high prevalence of $H$. pylori infections in institutions for people with intellectual disability. Health care workers working in these institutions are especially vulnerable because of their close contact [17].

H. pylori inhabits the gastric mucosa. Consequently, the endoscopes used to perform gastroscopies on patients affected become contaminated. Infection can be passed on to other patients via these endoscopes. The first recorded nosocomial infection with $H$. pylori was reported in 1979. 17 of 37 healthy subjects who took part in a study on acid concentrations in the stomach developed gastritis after an endoscopy [18]. Although, appropriate decontamination can certainly prevent transmission via this route $[18,19]$. Given that the oraloral route seems to be the predominant way of transmitting $H$. pylori, the question is whether doctors who perform gastroscopies, or the nurses who assist them, run an increased risk of infection. Several reviews have been published, but some data were controversial. We therefore checked the literature and conducted a metaanalysis to determine the occupational risk of $H$. pylori infection among gastroenterological personnel.

\section{Methods}

\section{Search strategy and screening}

For the literature search, we first considered the studies published in three review articles published in 1999
[20], 2001 [21] and 2004 [22]. This work was supplemented by a systematic search for literature in the MEDLINE and EMBASE databases using appropriate keywords - "Helicobacter pylori" combined with "occupational risk, endoscopy, gastroenterologist, healthcare worker" - for the years 1999 to 2010 . We also searched through the reference lists of the chosen studies and included appropriate publications in our work.

The criteria for inclusion related to the following:

- Study design: cohort study or cross-sectional study

- Study population: the study investigates gastroenterologists and/or their assistants (nurses)

- Exposure: the study investigates occupation as a risk factor

- Languages: German and English

\section{Study quality}

The methodological quality of the studies was assessed as moderate or good. A study was categorised as moderate if it did not take into account the potential confounding effect of age and socioeconomic status or did not adequately describe the control group's origin in order to check for the potential of confounding. Very small studies with fewer than 30 subjects per group also counted as methodologically moderate. Three authors carried out the literature screening and quality evaluation independently from one other and then compared their findings. Where they disagreed, a consensus was reached by means of discussion.

\section{Statistical analysis}

The studies are differentiated into prospective incidence studies and retrospective prevalence studies. For the purposes of statistical analysis, we used the information on the number of gastroenterological personnel and the control group as a whole, and the proportion in each case that had tested positive for $H$. pylori. This data was used to calculate prevalence ratios as effect estimates in the case of retrospective original studies. These are described as relative risks (RR). 95\% confidence intervals $(95 \% \mathrm{CI})$ were generated to serve as statistical tests. For the purpose of meta-analysis, a combined effect estimate was calculated using the Mantel-Haenszel method for dichotomous outcomes. Stratification enabled us to conduct further differentiated analyses relating to individual occupational groups, the kind of controls, the study region and the time of publication.

\section{Heterogeneity}

We carried out a chi-square test $\left(\chi^{2}\right)$ in order to examine the statistical heterogeneity between studies. If there was statistically significant heterogeneity $(\mathrm{P}<0.05)$ the random effect model was used to calculate the combined effect estimate, otherwise the fixed effect method was used. 


\section{Sensitivity analysis}

The impact of the studies on the combined effect estimate finding was tested by excluding individual studies from the analysis and examining the estimate stability [23]. The quality of the studies was also compared in this context. Where there is a clearly recognisable difference, the moderate studies can be considered separately or excluded from the analysis.

\section{Publication bias}

We first showed a possible publication bias graphically using a funnel plot. The effect estimate was plotted versus the precision of the estimate (defined as the inverse of standard error $\left.\left(1 / \mathrm{SE}_{\mathrm{i}}\right)\right)$. An asymmetry of this funnel plot indicates publication bias. The funnel plot asymmetry was additionally measured following a linear regression approach on the natural logarithmic scale of the effect estimate. In this method, the standard normal deviate, defined as the effect estimate divided by its standard error, is regressed against the precision. The intercept provides a measure of asymmetry. The greater the deviation from zero, the stronger the evidence of asymmetry [24].

The analyses were carried out using Review Manager (RevMan 5) and Microsoft Excel.

\section{Results}

\section{Studies identified and assessment of study quality}

24 studies [25-48] dealing with occupational exposure to H. pylori among gastroenterologists and their colleagues were identified and included in our meta-analysis. The individual studies are listed in Tables 1 and 2. In the main, the study design is cross-sectional, so prevalence ratios are stated. The study by Hildebrand [27] is an exception. Here, the longitudinal design enables the authors to show incidence as well as prevalence. Thus, an incidence of $2.6 \%$ per year was found among gastroenterologists and an incidence of $0.14 \%$ per year among the control group. However, only retrospective prevalence analyses were taken into account in the metaanalysis.

When study quality was assessed on the basis of the abovementioned criteria, 15 studies were categorised as good [25-36,46-48], and nine as methodologically moderate [37-45].

\section{Meta-analysis}

Pooled analysis of all 24 studies included showed that gastroenterological staff (RR 1.34; 95\%CI 1.14-1.58) exhibited a significantly increased risk of $H$. pylori infection. Pooling of all methodologically good studies confirmed this increased risk (RR 1.52; 95\%CI 1.27-1.81), whereas the methodologically moderate studies provided no evidence of higher risk.
A differentiated analysis of the studies, which drew a difference between the information on gastroenterologists, their nurses/assistants and gastroenterological personnel in general, produced a similar picture. While the studies as a whole and the methodologically good studies (Figure 1) showed statistically significant results, the moderate studies showed no difference in $H$. pylori prevalence between gastroenterological personnel and the controls (Table 3).

The studies chose to use different control groups, recruiting them either directly from the population at large or non-medical fields ( = without contact with patients, Table 1), or from hospital staff from other departments ( $=$ in contact with patients, Table 2 ). When the studies are stratified by non-medical controls, pooled analysis showed statistically significant risks for all personnel (RR 1.74; 95\%CI 1.23-2.48), for doctors (RR 1.39; 95\%CI 1.09-1.77) and for nurses/assistants (RR 1.37; 95\% CI 1.08-1.74). In contrast, comparison with medical controls revealed no statistically significant risks.

When the studies were differentiated by location in Europe, Asia and America/Australia, studies conducted in Asia showed a marked 50\% increase in risk for all occupational groups in gastroenterological departments. Assistants were the only group observed in the European studies to be at significantly higher risk statistically, whereas doctors were at significantly higher risk in America/Australia. If only methodologically good studies are considered, there is no change in the Asian studies, whereas in Europe a statistically significant increase in risk for all personnel can be seen.

Stratification by method of diagnosis revealed no striking differences between breath test diagnosis and diagnosis by serological examination. Stratification by date of publication showed significantly higher risks for the period 1990-1999 in all groups statistically, while no increase in risk could be seen prior to 1990 . For studies published in 2000 or later, statistically significant risks could be seen for endoscopy personnel in general, and for nurses/assistants.

\section{Heterogeneity and sensitivity analysis}

Testing for homogeneity revealed clear evidence of heterogeneity among most studies included in this paper. Accordingly, the random effect model was used to calculate the pooled effect estimate. A sensitivity analysis was also carried out, excluding individual studies from the meta-analysis one by one so as to show their influence on the pooled effect estimate. The relative risks thus calculated ranged from 1.26 to 1.40 and were all statistically significant.

\section{Publication Bias}

The funnel plot showed no evidence of a marked publication bias (funnel plot not shown), nor did linear 
Table 1 Information, risk estimation and quality assessment of studies with non-medical controls

\begin{tabular}{|c|c|c|c|c|c|c|c|}
\hline First author, year & Study area & $\begin{array}{l}\text { Gastros } \\
\text { (HP positive \%) }\end{array}$ & $\begin{array}{l}\text { Controls } \\
\text { (HP positive \%) }\end{array}$ & $\begin{array}{l}\text { Diagnostic } \\
\text { method }\end{array}$ & $\mathrm{RR}$ & $95 \% \mathrm{Cl}$ & $\begin{array}{l}\text { Study } \\
\text { quality }\end{array}$ \\
\hline Mastromarino 2005 [25] & Italy & $\begin{array}{l}\text { S } 92(40) \\
\text { D } 47(34) \\
\text { A } 45(37)\end{array}$ & 52 (19) hosp & Stool antigen test & $\begin{array}{ll}\text { S } 1.9 \\
\text { D } 1.8 \\
\text { A } 2.1\end{array}$ & $\begin{array}{l}1.04-3.6 \\
0.9-3.5 \\
1.1-4.0\end{array}$ & good \\
\hline Birkenfeld 2004 [26] & Israel & $\begin{array}{l}\text { S } 190(73) \\
\text { D } 88(72) \\
\text { A } 50(53)\end{array}$ & 4633 (53) pat & Breath test & $\begin{array}{ll}\text { S } 1.8 \\
\text { D } 1.4 \\
\text { A } 1.5\end{array}$ & $\begin{array}{l}1.3-1.5 \\
1.2-1.5 \\
1.3-1.7\end{array}$ & good \\
\hline Hildebrand 2000 [27] & Switzerland & D 92 (39) & 168 (38) pop & Breath test & D 1.03 & $0.8-1.4$ & good \\
\hline Ellett 1999 [28] & USA & A 138 (14) & 112 (18) don & Serology & A 0.8 & $0.4-1.4$ & good \\
\hline Monés 1999 [29] & Spain & D $137(53)$ & 189 (52) pop & Breath test & D 1.03 & $0.8-1.3$ & good \\
\hline Abbas 1998 [30] & Pakistan & $\begin{array}{l}\text { S } 33(79) \\
\text { D } 19(68) \\
\text { A } 14(93)\end{array}$ & $\begin{array}{l}33(58) \\
\text { neighbours }\end{array}$ & Serology & $\begin{array}{l}\text { S } 1.4 \\
\text { D } 1.2 \\
\text { A } 1.6\end{array}$ & $\begin{array}{l}0.97-1.9 \\
0.8-1.8 \\
1.2-2.2\end{array}$ & good \\
\hline Nishikawa 1998 [31] & Japan & $\begin{array}{l}\text { S } 121(30) \\
\text { D } 92(30) \\
\text { A } 29(28)\end{array}$ & 101 (25) pop & Serology & $\begin{array}{l}\text { S } 1.2 \\
\text { D } 1.2 \\
\text { A } 1.1\end{array}$ & $\begin{array}{l}0.8-1.9 \\
0.8-2.0 \\
0.6-2.2\end{array}$ & good \\
\hline Braden 1997 [32] & Germany & $\begin{array}{l}\text { S } 1091 \text { (38) } \\
\text { D } 922(38) \\
\text { A } 169 \text { (37) }\end{array}$ & 413 (27) pop & Breath test & $\begin{array}{ll}\text { S } 1.4 \\
\text { D } 1.4 \\
\text { A } 1.4\end{array}$ & $\begin{array}{l}1.2-1.7 \\
1.2-1.7 \\
1.1-1.7\end{array}$ & good \\
\hline Goh 1996 [33] & Malaysia & $\begin{array}{l}\text { S } 82(33) \\
\text { D } 34(41) \\
\text { A } 48(27)\end{array}$ & 53 (11) pop & Breath test & $\begin{array}{l}\text { S } 2.9 \\
\text { D } 3.6 \\
\text { A } 2.4\end{array}$ & $\begin{array}{l}1.3-6.6 \\
1.6-8.5 \\
0.99-5.8\end{array}$ & good \\
\hline Liu 1996 [34] & China & $\begin{array}{l}\text { S } 170(81) \\
\text { D } 125(82) \\
\text { A } 45(78)\end{array}$ & 702 (45) pop & Serology & $\begin{array}{ll}\text { S } 1.8 \\
\text { D } 1.9 \\
\text { A } 1.7 \\
\end{array}$ & $\begin{array}{l}1.6-2.0 \\
1.7-2.1 \\
1.5-2.1 \\
\end{array}$ & good \\
\hline Chong 1994 [35] & USA & $\begin{array}{l}\text { S } 122(53) \\
\text { D } 111(52) \\
\text { A } 11(52)\end{array}$ & 510 (14) don & Serology & $\begin{array}{l}\text { S } 3.8 \\
\text { D } 3.7 \\
\text { A } 3.9 \\
\end{array}$ & $\begin{array}{l}2.9-5.0 \\
2.8-4.9 \\
2.2-6.9 \\
\end{array}$ & good \\
\hline Lin 1994 [36] & Australia & $\begin{array}{l}\text { D } 39(69) \\
\text { A } 107(17) \\
\end{array}$ & $\begin{array}{l}195 \text { (37) pop } \\
115 \text { (37) pop } \\
\end{array}$ & Serology & $\begin{array}{l}\text { D } 1.9 \\
\text { A } 0.6 \\
\end{array}$ & $\begin{array}{l}1.4-2.5 \\
0.4-1.01 \\
\end{array}$ & good \\
\hline Velasco 2007 [37] & Cuba & S 38 (39) & 38 (8) hosp & Serology & S 5.0 & $1.6-15.9$ & moderate \\
\hline Prónai 2000 [38] & Hungary & D 101 (30) & 426 (54) pop & Breath test & D 0.6 & $0.4-0.8$ & moderate \\
\hline Kamat 1999 [39] & India & D 17 (29) & 35 (20) hosp & Serology & D 1.5 & $0.6-4.0$ & moderate \\
\hline Rudi 1997 [40] & Germany & S 75 (24) & 110 (35) hosp & Serology & S 0.7 & $0.4-1.1$ & moderate \\
\hline Pristautz 1994 [41] & Austria & D 88 (57) & 100 (51) pop+don & Serology & D 1.1 & $0.9-1.5$ & moderate \\
\hline Mitchel 1989 [42] & Australia & $\begin{array}{l}\text { S } 101(30) \\
\text { D } 33(51) \\
\text { A } 68(19)\end{array}$ & 715 (22) don & Serology & $\begin{array}{l}\text { S } 1.4 \\
\text { D } 2.4 \\
\text { A } 0.9\end{array}$ & $\begin{array}{l}0.99-1.9 \\
1.7-3.4 \\
0.5-1.5\end{array}$ & moderate \\
\hline Reiff 1989 [43] & Germany & S $45(69)$ & $\begin{array}{l}165 \text { (65) } \\
\text { stud+don+pat }\end{array}$ & Serology & S 1.1 & $0.9-1.3$ & moderate \\
\hline Rawles 1987 [44] & USA & S $38(32)$ & 20 (10) don & Serology & 53.2 & $0.8-12.8$ & moderate \\
\hline Morris 1986 [45] & New Zealand & $\begin{array}{lll}\text { S } 36 & (25) \\
\text { D } 21(33) \\
\text { A } 11 & (18)\end{array}$ & 261 (37) pop & Serology & $\begin{array}{l}\text { S } 0.7 \\
\text { D } 0.9 \\
\text { A } 0.5\end{array}$ & $\begin{array}{l}0.4-1.2 \\
0.5-1.7 \\
0.1-1.8\end{array}$ & moderate \\
\hline
\end{tabular}

Gastros = gastroenterological staff.

$\mathrm{S}=$ staff, $\mathrm{D}=$ doctors, $\mathrm{A}=$ assistants, don = blood donors, pat = patients, stud = students.

hosp $=$ hospital staff $=$ without contact with patients or not defined.

med $=$ medical staff $=$ in contact with patients.

regression reveal any significant funnel plot asymmetry (intercept 0.59; 95\%CI -1.95-1.83).

\section{Discussion}

Pooled analysis of 24 retrospective prevalence studies revealed a higher risk of $H$. pylori infections among endoscopy personnel, which was statistically significant. This risk is evident for gastroenterologists and for their assistants. The composition of the control group was also found to have a considerable impact. The inclusion of controls that were from the general population or were at least non-medical highlighted significant 
Table 2 Information, risk estimation and quality assessment of studies with medical controls

\begin{tabular}{|c|c|c|c|c|c|c|c|}
\hline First author, year & Study area & $\begin{array}{l}\text { Gastros } \\
\text { (HP positive \%) }\end{array}$ & $\begin{array}{l}\text { Controls } \\
\text { (HP positive \%) }\end{array}$ & $\begin{array}{l}\text { Diagnostic } \\
\text { method }\end{array}$ & RR & $95 \% \mathrm{Cl}$ & $\begin{array}{l}\text { Study } \\
\text { quality }\end{array}$ \\
\hline Noone 2006 [46] & Scotland & A 74 (32) & $148(33)$ & Serology & A 0.98 & $0.7-1.5$ & good \\
\hline Mastromarino 2005 [25] & Italy & $\begin{array}{l}\text { S } 92(40) \\
\text { D } 47(34) \\
\text { A } 45(37)\end{array}$ & $105(35)$ & Stool antigen test & $\begin{array}{ll}\text { S } 1.05 \\
\text { D } 0.97 \\
\text { A } 1.1 \\
\end{array}$ & $\begin{array}{l}0.7-1.5 \\
0.6-1.6 \\
0.7-1.8 \\
\end{array}$ & good \\
\hline Birkenfeld 2004 [26] & Israel & $\begin{array}{l}\text { S } 190(73) \\
\text { D } 88(72) \\
\text { A } 50(53)\end{array}$ & $98(70)$ & Breath test & $\begin{array}{ll}\text { S } & 1.02 \\
\text { D } & 1.00 \\
\text { A } & 1.02 \\
\end{array}$ & $\begin{array}{l}0.9-1.2 \\
0.9-1.2 \\
0.8-1.3 \\
\end{array}$ & good \\
\hline Monés 1999 [29] & Spain & D $137(53)$ & $44(50)$ & Breath test & D 1.07 & $0.8-1.3$ & good \\
\hline Potts 1997 [47] & England/Wales & D $30(50)$ & $30(10)$ & Breath test & D 5.0 & $1.6-15.5$ & good \\
\hline Braden 1997 [32] & Germany & $\begin{array}{l}\text { S } 1091 \text { (38) } \\
\text { D } 922(38) \\
\text { A } 169 \text { (37) }\end{array}$ & $604(36)$ & Breath test & $\begin{array}{l}\text { S } 1.05 \\
\text { D } 1.06 \\
\text { A } 1.02\end{array}$ & $\begin{array}{l}0.9-1.2 \\
0.9-1.2 \\
0.8-1.3\end{array}$ & good \\
\hline Su 1996 [48] & Taiwan & D $70(80)$ & $64(52)$ & Serology & D 1.6 & $1.2-2.0$ & good \\
\hline Goh 1996 [33] & Malaysia & $\begin{array}{l}\text { S } 82(33) \\
\text { D } 34(41) \\
\text { A } 48(27)\end{array}$ & $25(12)$ & Breath test & $\begin{array}{l}\text { S } 2.74 \\
\text { D } 3.4 \\
\text { A } 2.3 \\
\end{array}$ & $\begin{array}{l}0.9-8.3 \\
1.1-10.7 \\
0.7-7.2 \\
\end{array}$ & good \\
\hline Prónai 2000 [38] & Hungary & D $101(30)$ & $108(35)$ & Breath test & D 0.8 & $0.6-1.3$ & moderate \\
\hline Kamat 1999 [39] & India & D 17 (29) & $17(18)$ & Serology & D 1.7 & $0.5-5.9$ & moderate \\
\hline Rudi 1997 [40] & Germany & S $75(24)$ & $272(35)$ & Serology & S 0.7 & $0.5-1.1$ & moderate \\
\hline Mitchel 1989 [42] & Australia & $\begin{array}{l}\text { S } 101(30) \\
\text { D } 33(51) \\
\text { A } 68(19)\end{array}$ & 35 (29) & Serology & $\begin{array}{l}\text { S } 1.04 \\
\text { D } 1.8 \\
\text { A } 0.7\end{array}$ & $\begin{array}{l}0.6-1.9 \\
0.97-3.4 \\
0.3-1.4\end{array}$ & moderate \\
\hline
\end{tabular}

Gastros = gastroenterological staff.

$\mathrm{S}=$ staff, $\mathrm{D}=$ doctors, $\mathrm{A}=$ assistants.

differences in $H$. pylori prevalence. On the other hand, comparison with medical controls whose occupational exposure was characterised by contact with patients failed to confirm an increased risk for gastroenterological personnel.

Methodologically good studies were more likely to show statistically significant risks than studies of moderate quality. The use of inadequate controls or insufficient adjustment for confounders like age and socioeconomic status seemingly diluted the effect estimates in studies with moderate quality.

\section{Study area}

With respect to the study area, the question arises why the results showed clear levels of significance only in Asia, and not in Europe or America/Australia. The number of studies and their populations cannot account for this, given that more gastroenterological personnel were examined in Europe than in Asia (Table 4). Nor can the quality of studies be sufficient reason for this difference, although stratification by study quality did reveal larger effect estimates in methodologically good studies than in those with moderate quality in Europe. H. pylori prevalence in the present studies differs very widely as reflected in the individual study regions. The median prevalence in Europe is 39\% among doctors performing gastroscopies and $37 \%$ for their assistants. In contrast and somewhat surprisingly, the prevalence among non-medical control groups (studies $\mathrm{N}=8$ ) is $45 \%$, in contrast with the $35 \%$ prevalence among medical controls (studies $\mathrm{N}=7$ ). The prevalence among doctors and nurses in Asia is significantly higher by comparison (median 68\% and 78\% respectively), whereas among the control groups it is considerably lower (both $35 \%)$. There is also a clear difference between target population and controls in the studies from America/ Australia, albeit at a much lower level - doctors 52\%, assistants $18 \%$, non-medical controls $16 \%$, medical controls $29 \%$. The different $H$. pylori prevalence rates of the general population in different regions (Asia 50-80\%, Europe 30-50\%, North America 30\% [3]) corroborate the findings in the non-medical controls. However, why the present studies from Europe found a higher prevalence among non-medical controls than among gastroenterological personnel remains obscure.

\section{Diagnostic method}

In epidemiological studies, a $H$. pylori infection is mainly diagnosed by non-invasive methods such as serology or breath tests. More rarely, a stool antigen test is used. In the present studies this method was used to ascertain prevalence in only one study. In contrast, 16 studies used serological tests and seven used breath tests. After stratification by diagnostic method, the meta-analysis 
Gastroenterological staff

Abbas 1998
Birkenfeld 2004
Braden 1997
Chong 1994
Goh 1996
Liu 1996
Mastromarino 2005
Nishikawa 1998

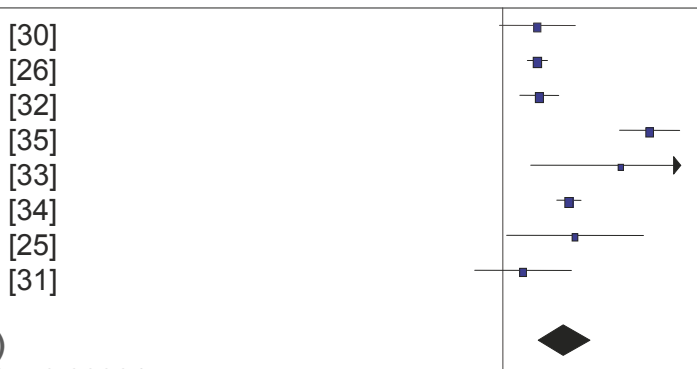

RR (95\% CI): 1.75 (1.39-2.20)

Heterogeneity: $\mathrm{Chi}^{2}=62.49, \mathrm{P}<0.00001$

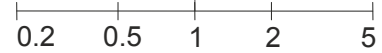

$\begin{array}{ll}\text { Gastroenterologists } & \\ \text { Abbas 1998 } & {[30]} \\ \text { Birkenfeld 2004 } & {[26]} \\ \text { Braden 1997 } & {[32]} \\ \text { Chong 1994 } & {[35]} \\ \text { Goh 1996 } & {[33]} \\ \text { Hildebrand 2000 } & {[27]} \\ \text { Lin 1994 } & {[36]} \\ \text { Liu 1996 } & {[34]} \\ \text { Mastromarino 2005 } & {[25]} \\ \text { Monés 1999 } & {[29]} \\ \text { Nishikawa 1998 } & {[31]} \\ \text { Potts 1997 } & {[47]} \\ \text { Su 1996 } & {[48]}\end{array}$

$\operatorname{RR}(95 \% \mathrm{Cl}): 1.62(1.33-1.97)$

Heterogeneity: $\mathrm{Chi}^{2}=85.69, \mathrm{P}<0.00001$

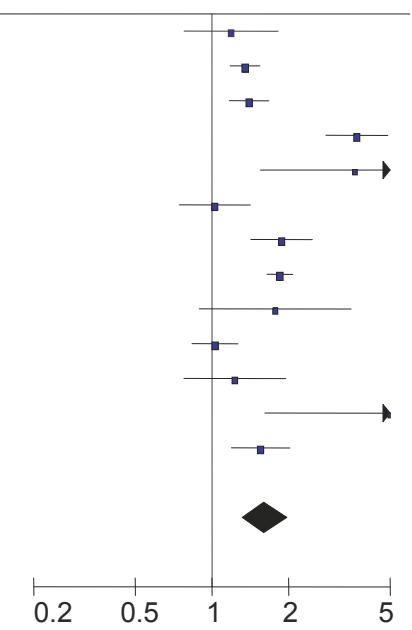

Gastroenterological assistants

\begin{tabular}{ll}
\hline Abbas 1998 & {$[30]$} \\
Birkenfeld 2004 & {$[26]$} \\
Braden 1997 & {$[32]$} \\
Chong 1994 & {$[35]$} \\
Ellett 1999 & {$[28]$} \\
Goh 1996 & {$[33]$} \\
Lin 1994 & {$[36]$} \\
Liu 1996 & {$[34]$} \\
Mastromarino 2005 & {$[25]$} \\
Nishikawa 1998 & {$[31]$} \\
Noone 2006 & {$[46]$}
\end{tabular}

RR (95\% Cl): $1.41(1.13-1.77)$

Heterogeneity: $\mathrm{Chi}^{2}=43.57, \mathrm{P}<0.00001$

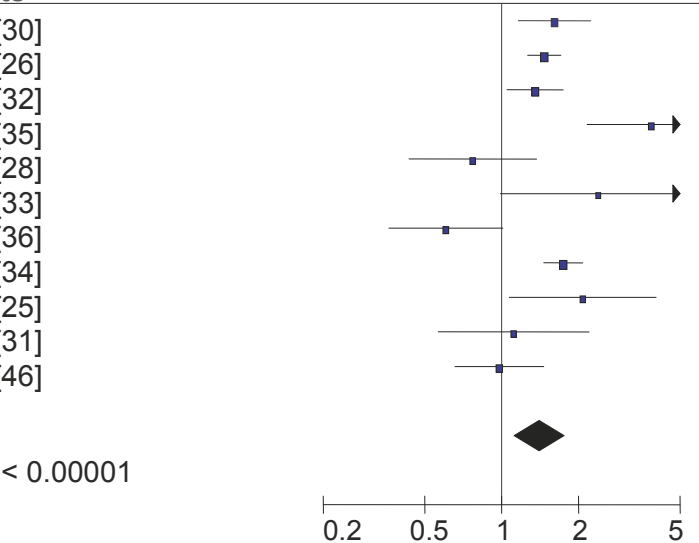

Figure 1 Forest plots of methodologically good studies for gastroenterological personnel and the risk of $H$. pylori infection. Block $=$ risk estimates, line $=95 \% \mathrm{Cl}$.

showed no differences in risk. The main advantage of serology is that it is a quick, low-cost method for use with large study populations. Nonetheless, this method has various limitations. These relate in part to defining a cut-off value to differentiate between positive and negative results and to the sensitivity of the test to changes in reagents and laboratory conditions, which is particularly relevant in longitudinal studies and repeat examinations. The breath test (C13-UBT) is somewhat more cost-intensive, but its sensitivity and specificity are 
Table 3 Summary of findings: Meta-analysis of H. pylori in gastroenterological personnel

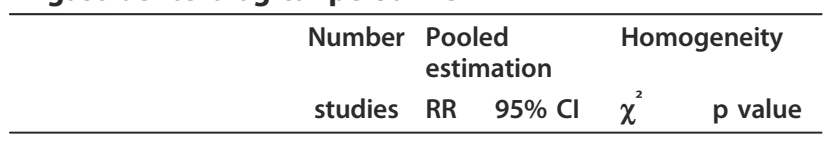

\begin{tabular}{|c|c|c|c|c|c|}
\hline All studies & & & & & \\
\hline All & 24 & 1.34 & $1.14-1.58$ & 174.51 & $<0.00001$ \\
\hline Staff & 14 & 1.51 & $1.23-1.84$ & 97.54 & $<0.00001$ \\
\hline Doctors & 18 & 1.48 & $1.22-1.81$ & 145.34 & $<0.00001$ \\
\hline Assistants & 13 & 1.33 & $1.06-1.66$ & 53.14 & $<0.00001$ \\
\hline
\end{tabular}

Good studies

\begin{tabular}{lccccc}
\hline All & 15 & 1.52 & $1.27-1.81$ & 100.00 & $<0.00001$ \\
Staff & 8 & 1.75 & $1.39-2.20$ & 62.49 & $<0.00001$ \\
Doctors & 13 & 1.62 & $1.33-1.97$ & 85.69 & $<0.00001$ \\
Assistants & 11 & 1.41 & $1.13-1.77$ & 43.57 & $<0.00001$
\end{tabular}

Moderate studies

\begin{tabular}{llllll}
\hline All & 9 & 1.04 & $0.77-1.41$ & 34.13 & $<0.00001$ \\
Staff & 6 & 1.16 & $0.79-1.69$ & 17.38 & 0.004 \\
Doctors & 5 & 1.12 & $0.62-2.03$ & 41.25 & $<0.00001$ \\
Assistants & 2 & 0.80 & $0.50-1.28$ & 0.72 & $0.4^{*}$
\end{tabular}

Medical controls

\begin{tabular}{lcclll}
\hline Staff & 6 & 1.02 & $0.90-1.15$ & 6.30 & $0.28^{*}$ \\
$\quad$ good studies & 4 & 1.06 & $0.96-1.18$ & 3.17 & $0.37^{*}$ \\
Doctors & 10 & 1.20 & $0.99-1.45$ & 23.58 & 0.005 \\
$\quad$ good studies & 7 & 1.21 & $0.98-1.50$ & 19.00 & 0.004 \\
Assistants & 6 & 1.04 & $0.91-1.20$ & 3.66 & $0.6^{*}$ \\
$\quad$ good studies & 5 & 1.07 & $0.93-1.23$ & 2.06 & $0.73^{*}$
\end{tabular}

Non-medical controls

\begin{tabular}{lccccc}
\hline Staff & 14 & 1.51 & $1.23-1.84$ & 97.54 & $<0.00001$ \\
$\quad$ good studies & 8 & 1.77 & $1.40-2.23$ & 63.90 & $<0.00001$ \\
Doctors & 16 & 1.41 & $1.13-1.77$ & 159.35 & $<0.00001$ \\
$\quad$ good studies & 11 & 1.55 & $1.22-1.96$ & 100.13 & $<0.00001$ \\
Assistants & 12 & 1.37 & $1.08-1.73$ & 48.13 & $<0.00001$ \\
$\quad$ good studies & 10 & 1.47 & $1.17-1.85$ & 37.89 & $<0.00001$
\end{tabular}

Study area Europe

\begin{tabular}{llllll}
\hline Staff & 4 & 1.16 & $0.85-1.59$ & 11.91 & 0.008 \\
$\quad$ good studies & 2 & 1.43 & $1.21-1.69$ & 0.97 & $0.33^{*}$ \\
Doctors & 7 & 1.13 & $0.85-1.51$ & 35.73 & $<0.00001$ \\
$\quad$ good studies & 5 & 1.30 & $0.99-1.72$ & 13.60 & 0.009 \\
Assistants & 3 & & & & \\
$\quad$ good studies & & 1.30 & $1.06-1.59$ & 3.95 & $0.14^{*}$
\end{tabular}

Study area Asia

\begin{tabular}{llllll}
\hline Staff & & & & & \\
$\quad$ good studies & 5 & 1.53 & $1.24-1.89$ & 19.33 & 0.0007 \\
Doctors & 7 & 1.53 & $1.26-1.86$ & 18.97 & 0.004 \\
$\quad$ good studies & 6 & 1.54 & $1.26-1.88$ & 18.94 & 0.002 \\
Assistants & & & & & \\
$\quad$ good studies & 5 & 1.58 & $1.40-1.78$ & 3.97 & $0.41^{*}$
\end{tabular}

Table 3 Summary of findings: Meta-analysis of H. pylori in gastroenterological personnel (Continued)

\begin{tabular}{lllllll}
\multicolumn{2}{l}{ Study area America / Australia } \\
\hline Staff (1 good,5 moderate) & & & & & & \\
Doctors & 6 & 2.07 & $0.98-4.40$ & 41.01 & $<0.00001$ \\
$\quad$ good studies & 4 & 2.08 & $1.30-3.34$ & 21.65 & $<0.00001$ \\
Assistants & 2 & 2.63 & $1.34-5.20)$ & 11.93 & 0.0006 \\
$\quad$ good studies & 5 & 0.99 & $0.47-2.10$ & 29.24 & $<0.00001$ \\
& 3 & 1.21 & $0.36-4.03$ & 28.02 & $<0.00001$
\end{tabular}

Diagnostic method: breath test

\begin{tabular}{llllll}
\hline Staff & 3 & 1.41 & $1.22-1.63$ & 3.46 & $<0.00001$ \\
Doctors & 7 & 1.23 & $0.92-1.64$ & 46.31 & $<0.00001$ \\
Assistants & 3 & 1.45 & $1.24-1.69$ & 1.55 & $0.46^{*}$
\end{tabular}

Diagnostic method: serology

\begin{tabular}{lccccc}
\hline Staff & 10 & 1.47 & $1.07-2.04$ & 84.87 & $<0.00001$ \\
Doctors & 11 & 1.66 & $1.33-2.09$ & 53.62 & $<0.00001$ \\
Assistants & 9 & 1.17 & $0.79-1.73$ & 57.54 & $<0.00001$
\end{tabular}

Publication date $\leq 1989$

\begin{tabular}{llllll}
\hline Staff & 4 & 1.14 & $0.94-1.38$ & 6.67 & $0.08^{*}$ \\
Doctors & 2 & 1.30 & $0.33-5.05$ & 15.56 & $<0.00001$ \\
Assistants & 2 & 0.80 & $0.50-1.28$ & 0.72 & $0.4^{*}$
\end{tabular}

Publication date 1990 - 1999

\begin{tabular}{lccccc}
\hline Staff & 7 & 1.62 & $1.17-2.24$ & 61.13 & $<0.00001$ \\
Doctors & 12 & 1.66 & $1.32-2.08$ & 80.05 & $<0.00001$ \\
Assistants & 8 & 1.42 & $1.02-1.98$ & 38.77 & $<0.00001$
\end{tabular}

Publication date $\geq 2000$

\begin{tabular}{llllll}
\hline Staff & 3 & 1.93 & $1.05-3.54$ & 6.87 & 0.03 \\
Doctors & 4 & 1.04 & $0.62-1.76$ & 36.22 & $<0.00001$ \\
Assistants & 3 & 1.36 & $1.15-1.61$ & 5.19 & $0.07^{*}$ \\
\hline
\end{tabular}

* Fixed effect model

in the order of $90-95 \%$, and this method is just as suitable as the stool test, particularly when examining children $[49,50]$.

\section{Comparison with other studies}

Since the $H$. pylori bacterium was discovered, numerous reviews have been published on the subject. Some dealt with the occupational risk of infection run by medical personnel in general, while some examined gastroenterological personnel in particular. Matysiak-Budnik [51] showed an association between occupational exposure and an increased risk of infection. Williams [20], too, stated that there were increased occupational risks for endoscopy personnel. However, the evidence in this review appears contradictory, since the findings varied between no risk and a five times greater risk. De Schryver et al. [21,22] were able 
Table 4 Regional distribution of studies

\begin{tabular}{|c|c|c|c|c|c|c|c|}
\hline $\begin{array}{l}\text { Study } \\
\text { area }\end{array}$ & $\begin{array}{l}\text { Number } \\
\text { studies }\end{array}$ & Quality & $\begin{array}{c}\text { Gastro staff } \\
\text { (HP positive \%) }\end{array}$ & $\begin{array}{c}\text { Doctors } \\
\text { (HP positive \%) }\end{array}$ & $\begin{array}{c}\text { Assistants } \\
\text { (HP positive \%) }\end{array}$ & $\begin{array}{l}\text { Non-medical controls } \\
\text { (HP positive \%) }\end{array}$ & $\begin{array}{c}\text { Medical controls } \\
\text { (HP positive \%) }\end{array}$ \\
\hline \multicolumn{8}{|c|}{ Europe } \\
\hline & 10 & all & $1303(37)$ & $1417(39)$ & $288(37)$ & $1623(45)$ & $1311(35)$ \\
\hline & 6 & good & $1183(37)$ & $1228(39)$ & $288(37)$ & $822(33)$ & $931(35)$ \\
\hline & 4 & moderate & $120(46)$ & $189(43)$ & & 801 (53) & $380(35)$ \\
\hline \multicolumn{8}{|c|}{ America / Australia } \\
\hline & 7 & all & $481(31)$ & $204(52)$ & $335(18)$ & $1929(16)$ & $35(29)$ \\
\hline & 3 & good & $268(42)$ & $150(61)$ & $256(17)$ & $895(16)$ & \\
\hline & 4 & moderate & $213(31)$ & $54(42)$ & $79(19)$ & $1034(16)$ & $35(29)$ \\
\hline \multicolumn{8}{|l|}{ Asia } \\
\hline & 7 & all & $596(73)$ & $445(68)$ & $186(78)$ & 5557 (35) & 204 (35) \\
\hline & 6 & good & $596(73)$ & $428(70)$ & $186(78)$ & $5522(45)$ & $187(52)$ \\
\hline & 1 & moderate & & $17(29)$ & & $35(20)$ & $17(18)$ \\
\hline
\end{tabular}

Gastro staff $=$ gastroenterological staff.

to show in their reviews increased risks for gastroenterologists and endoscopy personnel. Magalhaes Queiroz [52] found controversial data on the occupational risk, but they considered only some of the studies also included here for gastroenterological personnel.

\section{Strength and limitations of the review}

To our knowledge, this is the only meta-analysis to date to focus on the prevalence of $H$. pylori infection among endoscopy personnel. The strengths of this work are those of a meta-analysis. A meta-analysis can give a comprehensive overview of the state of research. Pooling different studies on a topic increases statistical power and validity in comparison with smaller individual studies and increases the likelihood of being able to identify actual differences that exist between groups [53]. Nonetheless, this form of study also has limitations, and the result of the effect estimate can only be considered and interpreted in relation to the underlying data. One problem in this paper is the statistical heterogeneity among studies. This was taken into account first by applying the random effect model. In addition, sub-group analyses were carried out so that only studies with identical characteristics were pooled and analysed [23].

The search strategy to listed papers might introduce publication bias, because inconclusive studies might be less likely published in listed journals. However, we controlled for publication bias and found no evidence for it.

An adequate control for confounding is crucial in occupational infectious disease epidemiology, especially when transmission of infection mainly occurs in childhood. Therefore, for a study to be classified as good, it needed to give the confounding nature of age and social status due consideration. However, the control for significant confounders via selection of the control group or by adjustment could not be assessed for effectiveness.
Although the assessment of study quality was taken into account, further criteria must be heeded when pooling and calculating pooled estimates - differences in risk factors, in the observance or adjustment of confounders, and in the study population (different regions, different survey dates, different study approaches/control groups). A lack of information about age, gender, ethnicity and work-related factors, such as the use of gloves and masks or the frequency of gastroscopies performed, precludes more differentiated analyses.

\section{Conclusions}

Gastroenterological personnel are exposed to an increased risk of $H$. pylori infection. Further studies involving suitable control groups and designed as prospectively as possible should identify the precise risk.

\section{Acknowledgements}

We wish to thank Dana Wendeler (BGW) for her support with the management of the literature. The study was conducted by the Institute for Health Service Research in Dermatology and Nursing, University Medical Center Hamburg-Eppendorf, with funding support from the Institution of Statutory Accident Insurance of the Health and Welfare Services (BGW). The BGW did not influence the study question or design, nor did it alter the analysis or interpretation of the results.

\section{Author details}

${ }^{1}$ University Medical Center Hamburg-Eppendorf, Institute for Health Service Research in Dermatology and Nursing, Hamburg, Germany. ${ }^{2}$ Occupational Health Division, Allergy and Clinical Immunology Division, Faculty of Medicine, Porto University, Porto, Portugal.

\section{Authors' contributions}

AS made substantial contributions to the design of the study and to the extraction and interpretation of data. She was involved in the drafting of the major scientific content in the manuscript. MH made substantial contributions to the analysis and interpretation of the data. She was involved in proofreading and editing the scientific content of the manuscript. CW made substantial contributions to the design of the study. She was involved in proofreading and editing the scientific content of the manuscript. JTC made substantial contributions to the interpretation of data 
and was involved in revising the manuscript critically for important intellectual content. AN made substantial contributions to the design and to the extraction and interpretation of data. He was involved in drafting the manuscript. CP made substantial contributions to the design of the study, the extraction of data, and to the analysis and interpretation of data. She was involved in drafting the manuscript. All authors confirm that they have seen and approved the final version and have no conflicts of interest.

\section{Competing interests}

The authors declare that they have no competing interests.

Received: 21 December 2010 Accepted: 31 May 2011

Published: 31 May 2011

\section{References}

1. Guarner J: The spectrum of gastric disease associated with Helicobacter pylori and other infectious gastritides. Curr Gastroenterol Rep 2004 6:441-446.

2. Mitchell HM: The epidemiology of Helicobacter pylori. Curr Top Microbiol Immunol 1999, 241:11-30.

3. Mandeville KL, Krabshuis J, Ladep NG, Mulder CJ, Quigley EM, Khan SA: Gastroenterology in developing countries: issues and advances. World J Gastroenterol 2009, 15:2839-2854.

4. Seher C, Thierfelder W, Dortschy R: Helicobacter pylori-prevalence in the German population. Gesundheitswesen 2000, 62:569-603.

5. Grimm W, Fischbach W: [Helicobacter pylori infection in children and juveniles: an epidemiological study on prevalence, socio-economic factors and symptoms]. Dtsch Med Wochenschr 2003, 128:1878-1883.

6. Marshall BJ, Warren JR: Unidentified curved bacilli in the stomach of patients with gastritis and peptic ulceration. Lancet 1984, 1:1311-1315.

7. Everhart JE: Recent developments in the epidemiology of Helicobacter pylori. Gastroenterol Clin North Am 2000, 29:559-578.

8. Brenner H, Arndt V, Bode G, Stegmaier C, Ziegler H, Stumer T: Risk of gastric cancer among smokers infected with Helicobacter pylori. Int J Cancer 2002, 98:446-449.

9. Moayyedi P, Axon AT, Feltbower R, Duffett S, Crocombe W, Braunholtz D, et al: Relation of adult lifestyle and socioeconomic factors to the prevalence of Helicobacter pylori infection. Int J Epidemiol 2002, 31:624-631.

10. Makristathis A, Pasching E, Schutze K, Wimmer M, Rotter ML, Hirschl AM: Detection of Helicobacter pylori in stool specimens by PCR and antigen enzyme immunoassay. J Clin Microbiol 1998, 36:2772-2774.

11. Banatvala N, Lopez CR, Owen R, Abdi Y, Davies G, Hardie J, et al: Helicobacter pylori in dental plaque. Lancet 1993, 341:380.

12. Dowsett SA, Kowolik MJ: Oral Helicobacter pylori: can we stomach it? Crit Rev Oral Biol Med 2003, 14:226-233.

13. Luman $\mathrm{W}$, Zhao $\mathrm{Y}, \mathrm{Ng}$ HS, Ling KL: Helicobacter pylori infection is unlikely to be transmitted between partners: evidence from genotypic study in partners of infected patients. Eur J Gastroenterol Hepatol 2002, 14:521-528.

14. Matsuda R, Morizane T: Helicobacter pylori infection in dental professionals: a 6-year prospective study. Helicobacter 2005, 10:307-311.

15. Lin SK, Lambert JR, Schembri MA, Nicholson L, Johnson $\mid H$ : The prevalence of Helicobacter pylori in practising dental staff and dental students. Aust Dent J 1998, 43:35-39.

16. Loster BW, Czesnikiewicz-Guzik M, Bielanski W, Karczewska E, Loster JE, Kalukin J, et al: Prevalence and characterization of Helicobacter pylori $(\mathrm{H}$. pylori) infection and colonization in dentists. J Physiol Pharmacol 2009, 60(Suppl 8):13-18

17. de Schryver A, Cornelis K, Van Winckel M, Moens G, Devlies G, Derthoo D, et al: The occupational risk of Helicobacter pylori infection among workers in institutions for people with intellectual disability. Occup Environ Med 2008, 65:587-591.

18. Nurnberg M, Schulz HJ, Ruden H, Vogt K: Do conventional cleaning and disinfection techniques avoid the risk of endoscopic Helicobacter pylori transmission? Endoscopy 2003, 35:295-299.

19. Wu MS, Wang JT, Yang JC, Wang HH, Sheu JC, Chen DS, et al: Effective reduction of Helicobacter pylori infection after upper gastrointestinal endoscopy by mechanical washing of the endoscope. Hepatogastroenterology 1996, 43:1660-1664.

20. Williams CL: Helicobacter pylori and endoscopy. J Hosp Infect 1999, 41:263-268.
21. De Schryver AA, Van Winckel MA: Helicobacter pylori infection: epidemiology and occupational risk for health care workers. Ann Acad Med Singapore 2001, 30:457-463.

22. De Schryver AA, Van Hooste WL, Van Winckel MA, Van Sprundel MP: Helicobacter pylori infection: a global occupational risk for healthcare workers? Int J Occup Environ Health 2004, 10:428-432.

23. Higgins JPT, Green S: Cochran Handbook for Systematic Reviews of Interventions 5.0.2. The Cochran Collaboration; 2009, updated September 2009.

24. Egger M, Davey Smith G, Schneider M, Minder C: Bias in meta-analysis detected by a simple, graphical test. BMJ 1997, 315:629-634.

25. Mastromarino P, Conti C, Donato K, Strappini PM, Cattaruzza MS, Orsi GB: Does hospital work constitute a risk factor for Helicobacter pylori infection? J Hosp Infect 2005, 60:261-268.

26. Birkenfeld S, Keter D, Dikman R, Shevah O, Shirin H, Niv Y: Prevalence of Helicobacter pylori infection in health-care personnel of primary care and gastroenterology clinics. J Clin Gastroenterol 2004, 38:19-23.

27. Hildebrand P, Meyer-Wyss BM, Mossi S, Beglinger C: Risk among gastroenterologists of acquiring Helicobacter pylori infection: casecontrol study. BMJ 2000, 321:149.

28. Ellett ML, Lou Q, Chong SK: Prevalence of immunoglobulin $\mathrm{G}$ to Helicobacter pylori among endoscopy nurses/technicians. Gastroenterol Nurs 1999, 22:3-6.

29. Mones J, Martin-de-Argila C, Samitier RS, Gisbert JP, Sainz S, Boixeda D: Prevalence of Helicobacter pylori infection in medical professionals in Spain. Eur J Gastroenterol Hepatol 1999, 11:239-242.

30. Abbas $Z$, Jafri W, Khan AH, Shah MA: Prevalence of Helicobacter pylori antibodies in endoscopy personnel and non-medical volunteers of Karachi. J Pak Med Assoc 1998, 48:201-203.

31. Nishikawa J, Kawai H, Takahashi A, Seki T, Yoshikawa N, Akita Y, et al: Seroprevalence of immunoglobulin $\mathrm{G}$ antibodies against Helicobacter pylori among endoscopy personnel in Japan. Gastrointest Endosc 1998, 48:237-243.

32. Braden B, Duan LP, Caspary WF, Lembcke B: Endoscopy is not a risk factor for Helicobacter pylori infection-but medical practice is. Gastrointest Endosc 1997, 46:305-310.

33. Goh KL, Parasakthi N, Ong KK: Prevalence of Helicobacter pylori infection in endoscopy and non-endoscopy personnel: results of field survey with serology and 14C-urea breath test. Am J Gastroenterol 1996, 91:268-270.

34. Liu WZ, Xiao SD, Jiang SJ, Li RR, Pang ZJ: Seroprevalence of Helicobacter pylori infection in medical staff in Shanghai. Scand J Gastroenterol 1996, 31:749-752.

35. Chong J, Marshall BJ, Barkin JS, McCallum RW, Reiner DK, Hoffman SR, et al: Occupational exposure to Helicobacter pylori for the endoscopy professional: a sera epidemiological study. Am J Gastroenterol 1994, 89:1987-1992.

36. Lin SK, Lambert JR, Schembri MA, Nicholson L, Korman MG: Helicobacter pylori prevalence in endoscopy and medical staff. J Gastroenterol Hepatol 1994, 9:319-324

37. Velasco Elizalde C, Fernandez Ferrer MA, Rodriguez Muniz N: [Serologic diagnosis of Helicobacter pylori in endoscopy personnel. Serology in endoscopists]. Rev Esp Enferm Dig 2007, 99:88-93.

38. Pronai $L$, Schandl L, Tulassay $Z$ : Prevalence of Helicobacter pylori infection in Hungarian gastroenterologists and general practitioners. Eur J Gastroenterol Hepatol 2000, 12:473-474.

39. Kamat AH, Mehta PR, Bhatia SJ, Koppikar GV: Seroprevalence of IgG antibodies to Helicobacter pylori among gastrointestinal endoscopists, radiologists and paramedical personnel. J Assoc Physicians India 1999, 47:866-868.

40. Rudi J, Toppe H, Marx N, Zuna I, Theilmann L, Stremmel W, et al: Risk of infection with Helicobacter pylori and hepatitis A virus in different groups of hospital workers. Am J Gastroenterol 1997, 92:258-262.

41. Pristautz $H$, Eherer $A$, Brezinschek $R$, Truschnig-Wilders $M$, Petritsch $W$, Schreiber F, et al: Prevalence of Helicobacter pylori antibodies in the serum of gastroenterologists in Austria. Endoscopy 1994, 26:690-696.

42. Mitchell HM, Lee A, Carrick J: Increased incidence of Campylobacter pylori infection in gastroenterologists: further evidence to support person-toperson transmission of C. pylori. Scand J Gastroenterol 1989, 24:396-400.

43. Reiff A, Jacobs E, Kist M: Seroepidemiological study of the immune response to Campylobacter pylori in potential risk groups. Eur J Clin Microbiol Infect Dis 1989, 8:592-596. 
44. Rawles JW, Harris ML, Paull G, Dick J, Yardley JH, Hendrix TR, et al: Antibody to Campylobacter pyloridis in endoscopy personnel, patients and controls. Abstr Pap 1987, 92:1589.

45. Morris A, Lloyd G, Nicholson G: Campylobacter pyloridis serology among gastroendoscopy clinic staff. N Z Med J 1986, 99:819-820.

46. Noone PA, Waclawski ER, Watt AD: Are endoscopy nurses at risk of infection with Helicobacter pylori from their work? Occup Med (Lond) 2006, 56:122-128.

47. Potts LF, Lewis SJ, Mountford RA: Prevalence of Helicobacter pylori in respiratory physicians performing bronchoscopy: a comparison with gastroenterologists using the carbon 13 urea breath test. Helicobacter 1997, 2:152-154

48. Su YC, Wang WM, Chen LT, Chiang W, Chen CY, Lu SN, et al: High seroprevalence of IgG against Helicobacter pylori among endoscopists in Taiwan. Dig Dis Sci 1996, 41:1571-1576.

49. Brown LM: Helicobacter pylori: epidemiology and routes of transmission. Epidemiol Rev 2000, 22:283-297.

50. Logan RP, Walker MM: $A B C$ of the upper gastrointestinal tract: Epidemiology and diagnosis of Helicobacter pylori infection. BMJ 2001, 323:920-922.

51. Matysiak-Budnik T, Megraud F: Epidemiology of Helicobacter pylori infection with special reference to professional risk. J Physiol Pharmacol 1997, 48(Suppl 4):3-17.

52. Magalhaes Queiroz DM, Luzza F: Epidemiology of Helicobacter pylori infection. Helicobacter 2006, 11(Suppl 1):1-5.

53. Ressing M, Blettner M, Klug SJ: Systematische Übersichtsarbeiten und Metaanalysen: Teil 6 der Serie zur Bewertung wissenschaftlicher Publikationen. Dtsch Arztebl 2009, 106:456-463.

\section{Pre-publication history}

The pre-publication history for this paper can be accessed here: http://www.biomedcentral.com/1471-2334/11/154/prepub

doi:10.1186/1471-2334-11-154

Cite this article as: Peters et al:: The occupational risk of Helicobacter pylori infection among gastroenterologists and their assistants. BMC Infectious Diseases 2011 11:154.

\section{Submit your next manuscript to BioMed Central and take full advantage of:}

- Convenient online submission

- Thorough peer review

- No space constraints or color figure charges

- Immediate publication on acceptance

- Inclusion in PubMed, CAS, Scopus and Google Scholar

- Research which is freely available for redistribution

Submit your manuscript at www.biomedcentral.com/submit 